\title{
Use of urinary bladder measurements from a point-of-care cysto-colic ultrasonographic view to estimate urinary bladder volume in dogs and cats
}

Gregory R. Lisciandro, DVM, DABVP, DACVECC and Geoffrey T. Fosgate, DVM, $\mathrm{PhD}, \mathrm{DACVPM}$

From Hill Country Veterinary Specialists, Spicewood, TX (Lisciandro) and the University of Pretoria, Department of Production Animal Studies, Onderstepoort, South Africa (Fosgate).

Please address all correspondence to: Dr. Gregory R. Lisciandro, Hill Country Veterinary Specialists, 1049 Lakeshore Drive, Spicewood, TX 78669. Email:

FastSavesLives@gmail.com

Offprints will not be available from the authors.

Conflicts of Interest: Dr. Lisciandro is the owner of FASTVet.com, a private corporation that provides veterinary ultrasound training to practicing veterinarians. He also teaches ultrasound courses for Sound, SonoSite, EI Medical, and scil animal care company, and has received ultrasound equipment on loan from SonoSite, Sound, EI Medical and scil animal care, and has licensed education materials to EI Medical.

Presented in abstract form at the 2015 International Veterinary Emergency and Critical Care Symposium, Washington D.C., September 2015. 


$\begin{array}{ll}\text { Abbreviations } \\ \text { AFAST } & \text { abdominal focused assessment with sonography for trauma } \\ \text { h } & \text { height } \\ \text { l } & \text { length } \\ \text { UOP } & \text { urine output } \\ \text { w } & \text { width }\end{array}$

\section{Abstract}

Objective: To develop a point-of-care ultrasound-derived formula to estimate urinary bladder volume non-invasively in dogs and cats.

Design: Prospective case series.

Setting: Private 24-hour veterinary emergency center.

Animals: Client-owned dogs and cats requiring urethral catheterization.

Interventions: Ultrasound measurements of length, width, and height of balloons filled with known water volumes were used to develop a formula to estimate urinary bladder volume using linear regression. The formula was then applied to point-of-care ultrasound-derived cysto-colic view measurements, and calculations were compared to total aspirated urine volume.

Measurements and Main Results: Fifteen balloons with known volumes (median, 126 $\mathrm{mL}$ [range, $27-689 \mathrm{~mL}$ ]) were used to identify length $\mathrm{x}$ width $\mathrm{x}$ height $(\mathrm{cm}) \mathrm{x} 0.2 \mathrm{x} \pi$ as the best formula to estimate urinary bladder volume in milliliters. Fourteen cats and 14 dogs were used for comparison of formula-derived volume estimate to actual urinary bladder volume. Median aspirated urine volume, bias (formula-derived minus actual 
aspirated), and percentage difference were $80 \mathrm{~mL},-4.1 \mathrm{~mL}$, and $-6.6 \%$ for cats, respectively. For dogs, the results were $78 \mathrm{~mL}, 3.4 \mathrm{~mL}$, and $3.6 \%$ respectively. Conclusions and Clinical Relevance: The point-of-care ultrasound-derived formula is useful to estimate urine volume non-invasively in dogs and cats.

Keywords: AFAST, canine, feline, ultrasound, urine output

\section{Introduction}

Urine output (UOP) can provide important clinical information for hospitalized dogs and cats because it guides IV fluid therapy and reflects kidney function when placed in clinical context. The measurement of UOP $(\mathrm{mL} / \mathrm{kg} / \mathrm{hr})$ can determine whether a dog or cat is producing acceptable volumes of urine. Urine output may be measured directly by the placement of a closed urinary collection system, by intermittent urethral catheterization and aspiration, or by free catch urine collection and measurement. Each method poses its own challenges and risks. For example, urethral catheterization is technically difficult in cats and female dogs. Sedation may be required, which carries risk especially in the critically or acutely ill patient. Furthermore, urinary bladder

catheterization can cause urethral trauma and iatrogenic urinary tract infection. ${ }^{1-4}$ Handling urine can expose staff members to drug metabolites and infectious diseases. ${ }^{1} \mathrm{~A}$ point-of-care ultrasound-based urinary bladder volume formula using simple linear measurements in both dogs and cats would be helpful to provide a safe, non-invasive, rapid, low-risk means of calculating UOP with minimal restraint. 
The purpose of this study was to develop a formula to estimate bladder volumes based on urinary bladder measurements acquired at the abdominal focused sonography for trauma (AFAST) cysto-colic view. The authors hypothesized that the formula would have acceptable bias relative to actual urinary bladder volume measurements in both dogs and cats.

\section{Materials and Methods}

Fifteen single-use, water-filled balloons ${ }^{\mathrm{a}}$ with a wide range of known volumes $(27-689$ $\mathrm{mL}$; median, $126 \mathrm{~mL}$ ) were placed in paper trays filled with acoustic coupling gel. Water balloons were chosen to develop the statistical model because the shape was considered similar to canine and feline urinary bladders and the true volume could be determined accurately. Maximum length (l) and height (h) were measured in centimeters using longitudinal (or sagittal) probe orientation; and maximum width (w) in centimeters by rotating the probe 90 degrees (transverse orientation), while mimicking the standard cysto-colic view used for AFAST examination. ${ }^{5}$ Acoustic coupling gel was used as contact medium. The study was approved by the institutional board of directors and subjects were enrolled with owner consent. Analogous measurements $(1, \mathrm{w}, \mathrm{h}$ in centimeters) were acquired at the AFAST cysto-colic view ${ }^{5}$ from male dogs and cats placed in right lateral recumbency (Figures 1,2). Care was taken to minimize probe pressure that could distort the shape of the urinary bladder, which could adversely affect measurements and calculations. ${ }^{6}$ All male dogs and cats underwent urethral catheterization for either urine collection for urinalysis or because of urethral obstruction. Urine was aspirated and total volume measured with complete urine aspiration confirmed 
using ultrasound after catheterization. Data including species, sex, body weight, reason for urethral catheterization, bladder measurements, and total urinary bladder volume in milliliters were recorded on standardized data sheets.

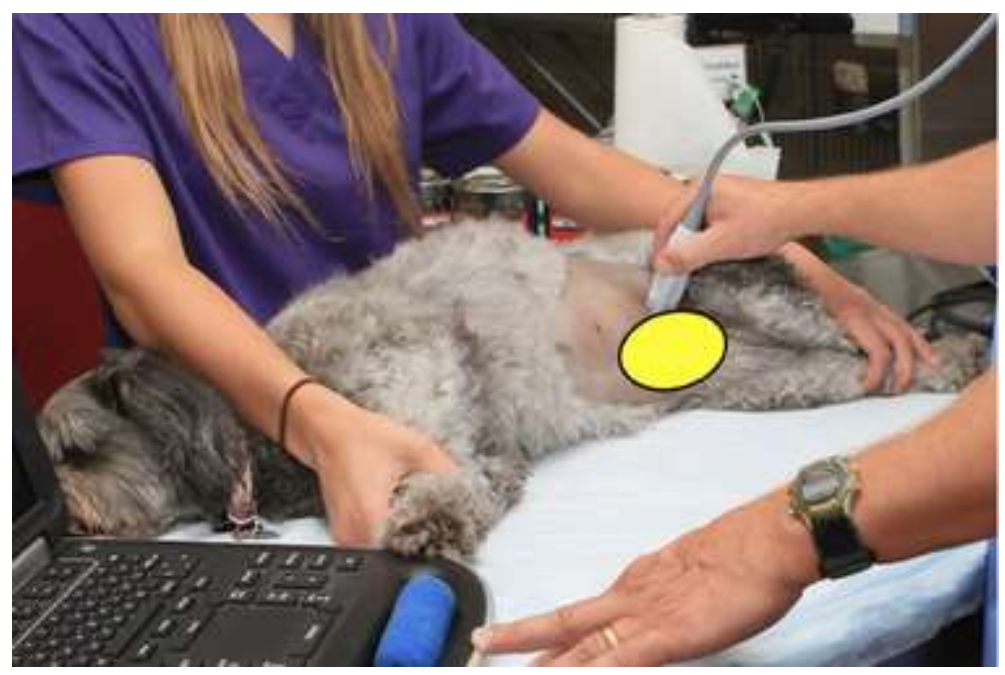

Figure 1. A dog in right lateral recumbency with an overlay of the urinary bladder (yellow oval). The white line indicates the cysto-colic pouch where the urinary bladder lies immediately against the gravity-dependent abdominal wall. The ultrasound probe shows the proper orientation of the ultrasound probe for the acquisition of the sagittal AFAST cysto-colic view and urinary bladder measurements. AFAST, abdominal focused assessment with sonography for trauma.
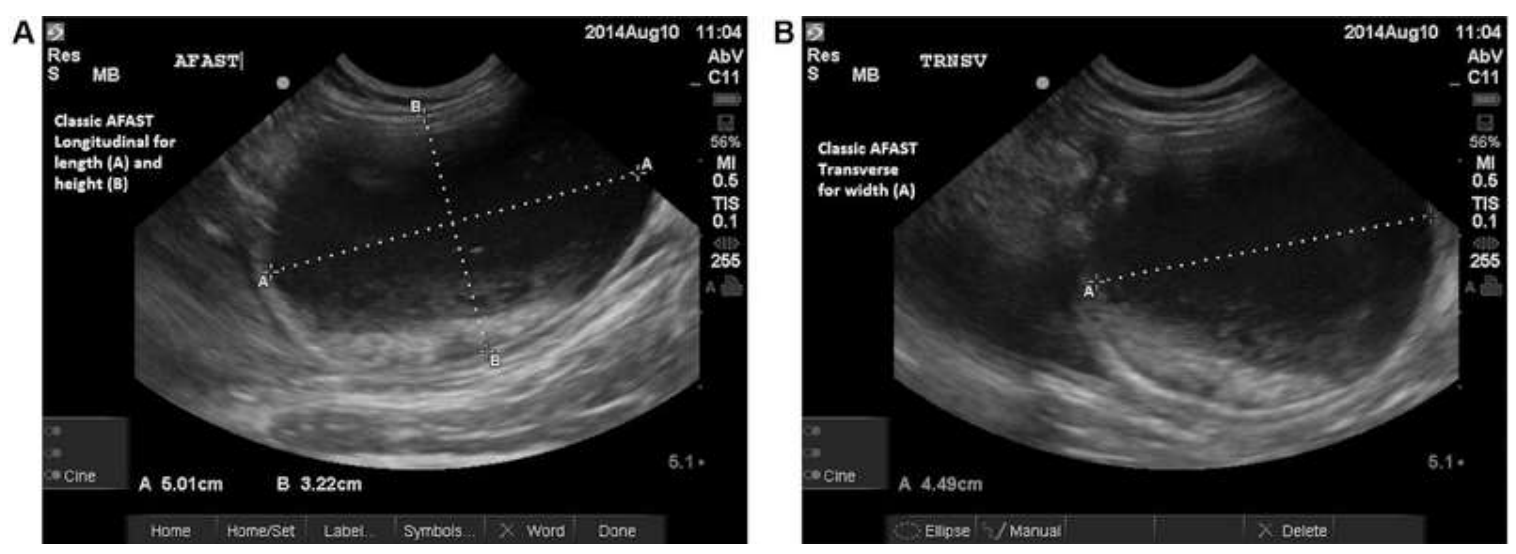

Figure 2. A) Ultrasound B-mode image showing urinary bladder at the AFAST cysto-colic view with the acquisition of the maximal length and height in longitudinal (sagittal) orientation (Cursor A represents length; Cursor B represents height). B) Ultrasound B-mode image showing urinary bladder at the AFAST cysto-colic view with the acquisition of the maximal width in transverse orientation (Cursor A represents width). AFAST, abdominal focused assessment with sonography for trauma. 


\section{Statistical Methods}

Simple linear regression was performed using water balloon data with the dependent variable being the true volume $(\mathrm{mL})$ of the balloon and the height $\mathrm{x}$ width $\mathrm{x}$ length $(\mathrm{cm}) \mathrm{x}$ $\pi$ as the predictor. The regression model did not include an intercept term. The predicted bladder volume was calculated for each animal using the estimated linear regression equation developed for the balloons. Bias was calculated as the predicted value - the aspirated volume from the bladder. Quantitative data were described using the median and range and were compared between cats and dogs using Mann-Whitney U tests. Modified Bland-Altman plots comparing predicted values to the aspirated volume (assumed as the true value rather than averaging predicted and aspirated values) were created. Statistical analysis was performed using commercially available software ${ }^{\mathrm{d}}$ and results were interpreted at the $5 \%$ level of significance.

\section{Results}

The linear regression using water balloons identified the best fitting equation for estimating bladder volume $(\mathrm{mL})$ as $1 \times \mathrm{h} \mathrm{x} \mathrm{w}(\mathrm{cm}) \times 0.2 \pi$. Fourteen adult male cats $(>1$ year) and 14 adult male dogs (> 1 year) were enrolled. Of the 14 cats, median body weight was $5.7 \mathrm{~kg}$ (range, $2.8-10.2 \mathrm{~kg}$ ) and median aspirated bladder volume was 80 $\mathrm{mL}$ (range, 30 - 147). All cats were catheterized due to urethral obstruction. Of the 14 dogs, median body weight was $13.9 \mathrm{~kg}$ (range, $3.6-36 \mathrm{~kg}$ ) and median aspirated bladder volume was $78 \mathrm{~mL}$ (range, $9-655 \mathrm{~mL}$ ). All dogs were catheterized for clinically indicated urinalysis. Based on the derived volume formula, the median (range) absolute bias was $-4.1 \mathrm{~mL}$ (range, $-30.1-14.9$ ) for cats and $-3.4 \mathrm{~mL}$ (range, $-123.8-81.2$ ) for 
dogs (Table 1, Figure 3). Overall, the mean percent bias was $-6.6 \%$ (range, $-26.2-19.6$ ) for cats $(\mathrm{N}=14)$ and $-3.6 \%$ (range, $-43.3-46.3)$ for dogs $(\mathrm{N}=14)$ though 3 dogs had a marked bias $>30 \%$. Of these 3 dogs, actual volumes of aspirated urine were large (264, 320 , and $655 \mathrm{~mL})$. The $95 \%$ limits of agreement for the percent bias were $-31.9 \%-$ $19.2 \%$ for cats and $-54 \%-45.1 \%$ for dogs. None of the 28 subjects had bladder luminal space-occupying lesions identified ultrasonographically.

Table 1. Comparison of the performance of the regression line for predicting bladder volume based on ultrasound measurements of the height, width, and length of the bladder between cats and dogs. Data are presented as the median (range).

\begin{tabular}{|l|l|l|l|}
\hline Variable & Cats $(\mathbf{n}=\mathbf{1 4})$ & Dogs $(\mathbf{n}=14)$ & $\boldsymbol{P}_{\text {-value }}^{*}$ \\
\hline Predicted volume, $\mathrm{mL}$ & $76.4(24.4-161.9)$ & $89.0(5.1-736.2)$ & 0.346 \\
\hline Aspirated volume, $\mathrm{mL}$ & $80.0(30.0-147.0)$ & $77.5(9.0-655.0)$ & 0.581 \\
\hline Bias, $\mathrm{mL}$ & $-4.1(-30.1-14.9)$ & $-3.4(-123.8-81.2)$ & 0.945 \\
\hline Bias, \% of actual & $-6.6(-26.2-19.6)$ & $-3.6(-43.3-46.3)$ & 0.241 \\
\hline
\end{tabular}

*Based on Mann-Whitney U tests. n, number.
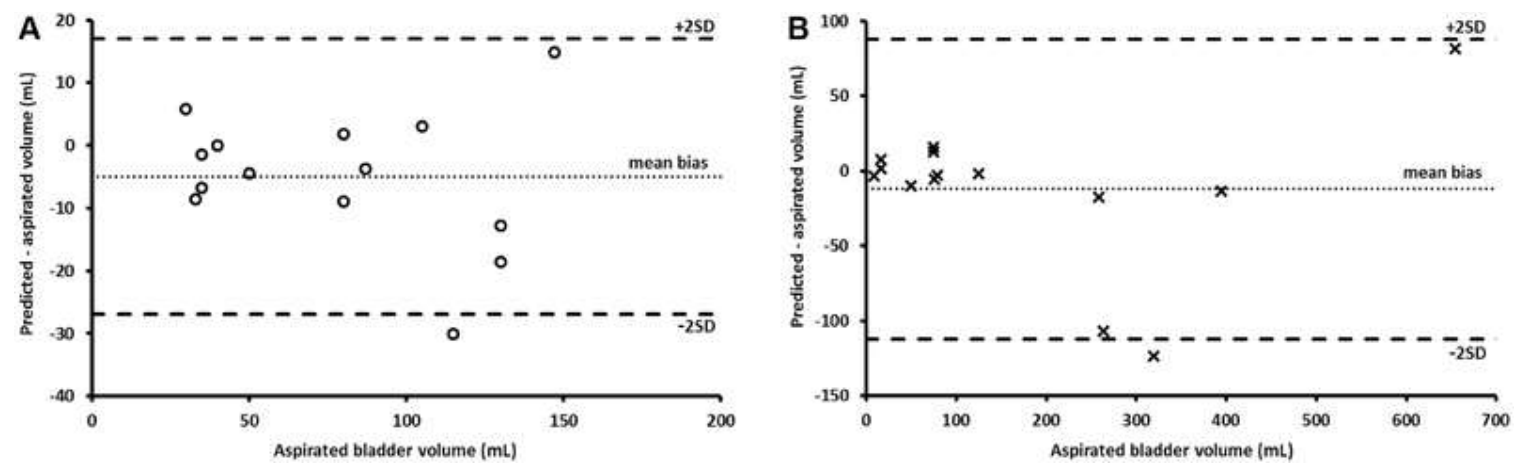

Figure 3. Modified Bland-Altman plots demonstrating the absolute bias in the linear regression predictive model for bladder urine volume (y-axis) against the volume of urine aspirated (x-axis) from 14 male cats (A) and 14 male dogs (B). Mean bias and $95 \%$ prediction interval is presented as $2 *$ standard deviation. SD, standard deviation. 


\section{Discussion}

The use of the simple, non-invasive urinary bladder formula of length $\mathrm{x}$ width $\mathrm{x}$ height $\mathrm{x}$ $0.2 \pi$ using urinary bladder measurements at the AFAST cysto-colic view provided an acceptable estimation of urinary bladder volume for 14/14 cats and 11/14 dogs. Similar 2-dimensional ultrasound formulas have been used for feline and canine gallbladder measurements ${ }^{7,8}$ and for urinary bladder measurements in men, women, children, and dogs primarily for detection of urine retention. ${ }^{1,9,10}$ To the authors' knowledge, a feline urinary bladder volume formula has not been previously evaluated.

Possible reasons for the 3 estimates with substantial bias (actual volumes of 264, 320 , and $655 \mathrm{~mL}$ ) include erroneous measurements of aspirated urine, weaknesses of the linear regression formula (less accurate at larger volumes),${ }^{10}$ errors or variability in the acquisition of ultrasound measurements, or inaccurate ultrasound confirmation of complete bladder emptying (when in fact residual urine remained undetected).

The formula proposed in the current study for cats, dogs, and overall tended to under-estimate urine volumes by a mean that was $<10 \%$ and similar to a previously published canine formula. ${ }^{10}$ Possibly the best use of such a formula would be its application in the calculation of UOP $(\mathrm{mL} / \mathrm{kg} / \mathrm{hr})$. Estimated urine volume using the current formula could prove helpful in monitoring dogs and cats after urine voiding as well as changes in bladder volume over time (a small bladder becoming larger with its volume difference calculated), although this was not directly studied. For example, if a $10 \mathrm{~kg}$ dog had point-of-care ultrasonographic cysto-colic view bladder measurements that estimated bladder volume at $120 \mathrm{~mL} 3$ hours after urine voiding, the dog's estimated UOP would be $4 \mathrm{~mL} / \mathrm{kg} / \mathrm{hr}$. If the formula under-estimated the true volume by a higher 
percentage of $15 \%$ (true volume was $138 \mathrm{~mL}$ ), then actual UOP would be $4.6 \mathrm{~mL} / \mathrm{kg} / \mathrm{hr}$ as opposed to the calculated $4 \mathrm{~mL} / \mathrm{kg} / \mathrm{hr}$. On the other hand, if the formula over-estimated urinary bladder volume by $15 \%$ (true volume was $102 \mathrm{~mL}$ ), actual UOP would be 3.4 $\mathrm{mL} / \mathrm{kg} / \mathrm{hr}$ as opposed to the calculated $4 \mathrm{~mL} / \mathrm{kg} / \mathrm{hr}$. All these calculations of UOP would be clinically acceptable for these relatively high UOPs, though this difference may be less clinically acceptable at lower UOPs. The current study's findings were similar to other veterinary studies by Atalan and colleagues who evaluated formulas with respect to urine retention and residual volumes in live and cadaver dogs. ${ }^{6,10}$

There are several limitations to the current study. Balloons were used to derive the formula with the assumption that they mimicked the shape of the canine and feline urinary bladder. All cats and dogs were males so sex differences could not be assessed. ${ }^{10}$ Due to small sample size, body weight was not evaluated, which may also affect formula calculations. ${ }^{10}$ All measurements were performed by the same sonographer (GRL) and thus inter-operator variability could not be determined. Moreover, sonographers performing bladder measurements should be aware that probe pressure can affect measured bladder dimensions, ${ }^{6}$ and thus affect the formula-derived urinary bladder volume. Furthermore, the measurement of length in longitudinal (sagittal) is approximated because the caudal aspect of the urinary bladder is often not fully visible (Figure 2A). It is also possible that in dogs and cats with known or suspected urinary tract disease as in the current study, altered bladder wall compliance, and thus altered bladder shape and measurements, could also adversely affect bladder volume estimation. Dogs and cats with neoplastic processes or other conditions that either distort the shape of the bladder or displace urine within the lumen of the urinary bladder would also be 
expected to have less accurate calculations. However, based on publications by Atalan and colleagues, changes in total urinary bladder volume in inflammatory and obstructive conditions (such as the current case population), and the use of live and cadaver dogs, are likely more theoretical than clinically relevant, unless luminal space-occupying lesions are present.

To fully evaluate the clinical utility of the current formula, larger numbers of dogs and cats of both sexes with variable urinary bladder volumes would be needed. The small sample size did not allow for the evaluation of potential covariates that might have affected model accuracy such as breed, body condition, and weight. The linear regression was also performed on data collected from balloons of specified sizes and the distribution was therefore non-normal, a typical assumption of linear regression. The regression model also deviated from the traditional regression approach because an intercept term was not included. The definition of the intercept is the value of the outcome when the predictor variables are 0 . An object with a 0 for the height, length, or width should have 0 volume. The inclusion of an intercept would have improved model fit for the analyzed data but would likely make the model less accurate when used clinically on a different population of animals. Moreover, the current model is similar with what has been published for dogs,${ }^{10}$ and despite its limitations the authors believe its simplicity by acquisition of the 3 linear measurements using a simple point-of-care ultrasonographic view will serve as a non-invasive, non-painful, safe, rapid, point-of-care method to estimate canine and feline urinary bladder volume. The bias in predictions was compared between cats and dogs and these initial results suggest that the model might be equally accurate in both species but our sample size was small and 4 dogs had substantially larger 
bias $(>30 \%)$ at very low volumes $(\leq 16 \mathrm{~mL})$ and very large volumes $(\geq 264 \mathrm{~mL})$. Thus, the current formula appears most accurate when used for the middle ranges of urinary bladder volumes in both dogs and cats.

Lastly, there are several points of note. Radiographic measurements and ultrasound-derived cross-sectional measurements have been previously studied. ${ }^{6,11}$ These radiographic and ultrasonographic formulas are not only more complex, and crosssectional ultrasound measurements more technically challenging, but neither provided clear clinically-relevant advantages over simple linear measurements. ${ }^{6,11}$ It also has been shown that 2-dimensional ultrasound measurements are inferior to 3-dimensional measurements ${ }^{4}$; however, 3 -dimensional ultrasound is not only limited in availability but also may not provide any clinically-relevant advantage over simple linear formulas. Positioning comparisons between dorsal and lateral recumbency regarding urinary bladder volume have also been performed. ${ }^{12}$ However, despite dorsal recumbency providing more accuracy, dorsal recumbency may be dangerous for hemodynamically fragile dogs and cats due compression of the caudal vena cava and aorta by the weight of abdominal organs, and by adverse effects on ventilation. ${ }^{5}$

In conclusion, the use a simple, point-of-care ultrasonographic cysto-colic view for estimation of urinary bladder volumes using the current simple equation carries the potential to be clinically useful for non-invasively estimation of urinary bladder volume and thus calculation of urine output in both dogs and cats. 


\section{Acknowledgements}

The authors thank the nursing staff at the Emergency Pet Center, Inc., including Emily Chavez, Michelle Crothers-Hines, Samantha Tierney, Doris Taffner, and Pam West; and Dr. Maria Hey, DVM, of Deerfield Animal Hospital, San Antonio, Texas for illustrative work.

\section{Footnotes}

${ }^{\mathrm{a}}$ Water Splashers Team Tubez Water Balloons, Imperial Toy, LLC, North Hills, CA ${ }^{\mathrm{b}}$ SonoSite Edge, SonoSite, Inc., Bothell, WA

${ }^{\mathrm{c}}$ Animal Intelligence, Animal Intelligence Software, Inc., Port Orchard, WA

${ }^{\mathrm{d}}$ MINITAB Statistical Software, Release 13.32, Minitab Inc, State College, PA

\section{References}

1. Gyampoh B, Crouch N, O'Brien P, et al. Interpartum ultrasound estimation of total bladder volume. BJOG 2004;111:103-108.

2. Milling TJ, Van Amerongen R, Melville L, et al. Use of ultrasonography to identify infants for whom urinary catheterization will be unsuccessful because of insufficient urine volume: validation of the urinary bladder index. Ann Emerg Med 2005;45(5):511513.

3. Byun S, Kim HH, Lee E, et al. Accuracy of bladder volume determination by ultrasonography: Are they accurate over entire bladder volume range? Adult Urology 2003;62:656-660. 
4. Bozsa S, Pto L, Bodis J, et al. Assessment of postoperative post void residual bladder volume using three-dimensional ultrasound volumetry. Ultrasound Med Biol 2011;37(4):522-529.

5. Lisciandro GR, Lagutchik MS, Mann KA, et al. Evaluation of abdominal fluid scoring system determined using abdominal focused assessment with sonography for trauma in 101 dogs with motor vehicle trauma. J Vet Emerg Crit Care 2009;19(5):426-437.

6. Atalan G, Barr FJ, Holt PE. Estimation of bladder volume using ultrasonographic determination of cross-sectional areas and linear measurements. Vet Radiol Ultrasound 1998;39(5):448-50.

7. Finn-Bodner ST, Park RD, Tyler JW, et al. Ultrasonographic determination, in vitro and in vivo, of canine gallbladder volumes, using four volumetric formulas and stepwiseregression models. Am J Vet Res 1993;54:832-835.

8. Penninck DG, Brisson JO, Webster C. Sonographic Assessment of Gallbladder Volume in Normal Cats. Vet Radiol Ultrasound 2010;51(6):665-666.

9. Dicuio M, Pomara G, Menchini F, et al. Measurements of urinary bladder volume: a comparison of five ultrasound calculation methods in volunteers. Arch Ital Adrol 2005:77(1):60-62.

10. Atalan G, Barr FJ, Holt PE. Assessment of urinary bladder volume in dogs by use of linear ultrasound measurements. Am J Vet Res 1998;59(1):10-15.

11. Atalan G, Barr FJ, Holt PE. Comparison of ultrasonographic and radiographic measurements of bladder dimensions and volume determinations. Res Vet Sci 1999;66:175-177.

12. Atalan G, Barr FJ, Holt PE. Effect of body position on ultrasonographic estimations of bladder volume. J Small Anim Pract 1999;40(4):177-79. 